\title{
CONTROLE PREDITIVO VIA MPC PARA DISTRIBUIÇÃO OTIMIZADA DE GASES COMBUSTÍVEIS*
}

Eduardo Oliveira Magalhães ${ }^{1}$ Marcelo Versiani Carneiro²

\section{Resumo}

Otimizar o consumo dos insumos energéticos e coprodutos é uma tarefa de suma importância na busca de competitividade das usinas siderúrgicas. O gás de alto forno é um importante componente dessa matriz energética e a maximização do seu consumo reduz sensivelmente os custos com a compra de outros combustíveis. Nesse cenário, o controle de nível dos gasômetros, equipamentos existentes nas siderúrgicas que exercem um papel fundamental na distribuição desse gás, não é trivial. Distúrbios e variações entre geração e consumo dificultam o desafio de manter a segurança operacional e ao mesmo tempo otimizar o consumo. Procedimentos operacionais e técnicas convencionais de controle não são suficientes para evitar perdas com a queima desse gás nas tochas de segurança (flares) dos altos fornos. Esse trabalho apresenta um caso real do uso de técnicas de controle preditivo MPC (Model Predictive Control) e regulatório para a distribuição em tempo real do gás de alto forno para os principais consumidores na Vallourec Tubos do Brasil em Belo Horizonte.

Palavras-chave: Gasômetro; Controle de nível; Gás de alto forno; Controle preditivo.

\section{PREDICTIVE CONTROL USING MPC FOR OPTIMUM FUEL DISTRIBUITION Abstract}

Optimize the consumption of energy inputs and byproducts are a very important target in terms of competitiveness for steel mills. The blast furnace gas is an important component of this energy matrix and maximize its consumption significantly reduces the costs of purchasing other fuels. In this scenario, gasholder's of steel making plants play a key role in the distribution of this gas. However, the level control and the gas distribution in these devices are not trivial. Disturbances and unbalances between generation and consumption increase the challenge of operating and at the same time optimize the consumption with safety. Operating procedures and conventional control techniques are not sufficient to avoid losses by burning this gas in the blast furnaces' torches (flares). This paper presents a real case of using predictive control techniques MPC (Model Predictive Control) and a regulatory control to distribute in real time the blast furnace gas to major consumers in Vallourec Tubos do Brasil in Belo Horizonte.

Keywords: Gasholder; Level control; Blast furnace gas; Predictive control.

1 Engenheiro Eletricista, MSc, Superintendência de Tecnologia de Projetos e Manutenção, Vallourec Tubos do Brasil, Belo Horizonte, MG.

2 Engenheiro de Controle e Automação, Superintendência de Tecnologia de Projetos e Manutenção, Vallourec Tubos do Brasil, Belo Horizonte, MG.

\footnotetext{
* Contribuição técnica ao $18^{\circ}$ Seminário de Automação e TI Industrial, 23 a 26 de setembro de 2014, São Paulo, SP, Brasil.
} 


\section{INTRODUÇÃO}

\subsection{Descrição do processo e problema}

A indústria de fabricação do aço é intensiva no uso de energia. Nesse segmento, a energia corresponde a cerca de $20 \%$ de todos os custos operacionais $[1,2]$. Com uma cadeia de produção grande e a geração de subprodutos que podem ser utilizados como fonte de energia para outras partes do processo, o uso e o consumo de energia nessa indústria não podem ser estudados isoladamente por cada unidade. A otimização do uso de energia requer o uso de sistemas computacionais, segundo Akimoto [3].

O gás de alto forno (GAF) é um subproduto do processo de redução do minério de ferro nos altos fornos. É um gás de baixo valor energético, mas mesmo assim, útil como substituto de outras fontes de energia como o gás natural (GN). Nas usinas siderúrgicas, diversas partes do processo utilizam o GAF: os próprios altos fornos no preaquecimento do ar utilizado nas ventaneiras, fornos de reaquecimento das linhas de laminação a quente, unidades de geração própria de energia elétrica, entre outros. A Figura 1 descreve de forma simplificada o circuito de produção e consumo de GAF na Vallourec Tubos do Brasil (VBR).

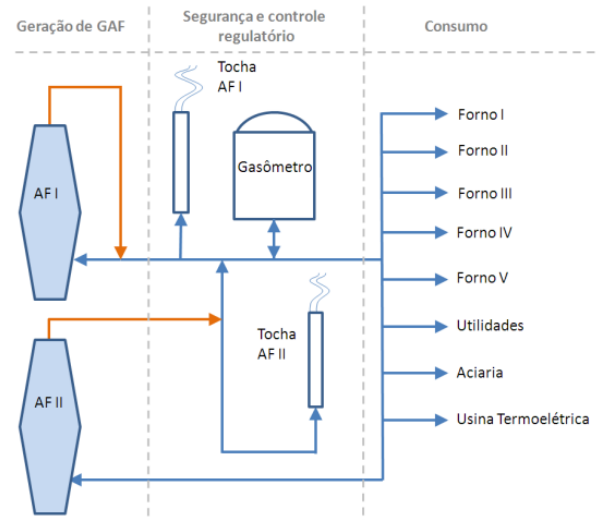

Figura 1. Fluxograma de geração e consumo de GAF na VBR. Fonte: VBR.

A geração e consumo de GAF é irregular e insere perturbações e desbalanceamento nesse circuito. Os desequilíbrios entre a produção e consumo do GAF podem ser observados nas variações do nível de um gasômetro.

Um gasômetro é um reservatório que funciona por meio de uma tampa móvel, chamada de disco ou teto flutuante, que mantém o GAF do circuito a uma pressão controlada. A posição do teto flutuante (ou nível do gasômetro) determina o volume de GAF disponível nesse reservatório [4]. O gasômetro da VBR é um reservatório de GAF, com volume útil de $20.000 \mathrm{~m}^{3}$.

Nível alto do gasômetro indica excesso de GAF no circuito. Por segurança operacional, esse excesso tem que ser queimado nas tochas dos altos fornos. Nesse momento, energia é perdida. Por outro lado, um nível baixo indica falta de GAF. Assim, é necessário reduzir o consumo, para evitar danos à estrutura do gasômetro.

Os métodos para otimizar a distribuição de GAF e outros combustíveis das usinas siderúrgicas existentes no mundo vem crescendo gradativamente. Uma prática comum é o desenvolvimento de modelos de programação inteira mista linear (MILP - Mixed Integer Linear Programming), o qual tem o objetivo de achar uma combinação ótima de distribuição de combustíveis de uma usina siderúrgica,

* Contribuição técnica ao $18^{\circ}$ Seminário de Automação e TI Industrial, 23 a 26 de setembro de 2014, São Paulo, SP, Brasil. 
levando em consideração o controle balanceado de nível de gasômetro. $\mathrm{O}$ artigo de Akimoto [3] foi o pioneiro nesse tipo de aplicação e onde se tem o primeiro registro de utilização desse modelo em uma usina siderúrgica no Japão. Em 2003, Kim e colaboradores $[5,6]$ também desenvolveram um otimizador baseado no modelo de Akimoto, porém considerando variáveis discretas, como por exemplo, a quantidade de queimadores que podem ser ligados e desligados das caldeiras de vapor. Já em 2010, Kong e colaboradores [1,2] utilizam otimização multiobjetivo para tentarem encontrar uma combinação mais otimizada de distribuição de combustíveis. Em 2011, Cardoso e colaboradores [7] desenvolveram um software que contém um otimizador para diversos combustíveis e a distribuição ótima para diversos gasômetros.

A utilização desses modelos de otimização de consumo de combustíveis já é considerada uma evolução, caso a distribuição de combustíveis seja feita de forma manual e baseada em planos de produção [8]. Entretanto, muitas das vezes, somente a predição da melhor combinação de onde distribuir o GAF ou outro subproduto, pode resultar em ganhos não tangíveis, pelo fato de essa distribuição não ser automática e dependente de intervenção humana. Observou-se que, na maioria dos trabalhos pesquisados e citados nesse artigo, não há de fato um algoritmo de controle atuando no processo, que possa fazer com que a predição ótima de consumo seja de fato garantida por um setpoint de vazão de gás em um consumidor (por exemplo, um forno ou uma caldeira).

Este trabalho trata do desenvolvimento de um sistema integrado para a distribuição automática e em tempo real do volume de GAF (excesso ou falta) na VBR. Esse sistema utiliza um controlador preditivo via modelo (MPC - model predictive controller) e tem como objetivo a redução das perdas de energia sem por em risco a segurança operacional do circuito de GAF. O objetivo desse controlador é predizer o nível do gasômetro da VBR e enviar setpoints ótimos de consumo de GAF para três consumidores da usina.

\subsection{Controle Preditivo MPC}

O controle preditivo baseado em modelo se refere a um tipo de controlador avançado, o qual utiliza um modelo (ou uma matriz de modelos) matemático para predizer a resposta futura de um processo [9]. Em cada intervalo de amostragem, o algoritmo de controle multivariável do MPC tenta otimizar o comportamento futuro do processo, calculando uma sequência futura de movimentos nas variáveis manipuladas (usualmente, setpoints de malhas PID).

Segundo Qin [9], o MPC foi inicialmente desenvolvido com o objetivo de suprir necessidades específicas de processos como refinarias de petróleo e termoelétricas na década de 70. Entretanto, atualmente é possível encontrar esse tipo de controlador nas mais diversas indústrias: química, alimentos, automotiva e aeroespacial.

As indústrias de refinaria e petroquímica ainda lideram a quantidade de aplicações de MPC com modelos lineares. A Tabela 1 mostra o quantitativo de aplicações por cada fabricante de software MPC disponível no mercado.

\footnotetext{
* Contribuição técnica ao $18^{\circ}$ Seminário de Automação e TI Industrial, 23 a 26 de setembro de 2014,
} São Paulo, SP, Brasil. 


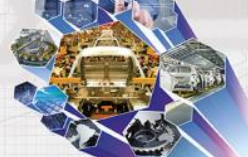

Tabela 1. Quantitativo de aplicações MPC por tipo de indústria/fabricante [10]

\begin{tabular}{|c|c|c|c|c|c|c|}
\hline Indústria/Solução MPC & Aspen Tech & $\begin{array}{c}\text { Honeywell } \\
\text { Hi-Spec }\end{array}$ & Adersa & PCL & SGS & Total \\
\hline Refinaria & 1200 & 480 & 280 & 25 & & 1985 \\
\hline Petroquímica & 450 & 80 & & 20 & & 550 \\
\hline Química & 100 & 20 & 3 & 21 & & 144 \\
\hline $\begin{array}{c}\text { Papel e Celulose } \\
\text { Are Gás }\end{array}$ & 18 & 50 & & & 68 \\
\hline Utilidades & & 10 & & & & 10 \\
\hline Mineração/Siderurgia & 8 & 10 & & 4 & 16 & 14 \\
\hline Alimentos & & 6 & 7 & 10 & & 37 \\
\hline Polímeros & 17 & & 41 & 10 & 17 \\
\hline Fornos & & & 42 & 3 & & 45 \\
\hline Aeroespacial & & & 13 & & & 13 \\
\hline Automotiva & & & & 7 & 450 & 1601 \\
\hline Não Classificada & 40 & 40 & 1045 & 26 & 450 & 4542 \\
\hline Total & 1833 & 696 & 1431 & 132 & 450 \\
\hline
\end{tabular}

Com base nos dados apresentados na Tabela 1, nota-se uma pequena quantidade de aplicações na indústria metalúrgica. Mesmo após pesquisas exaustivas em importantes bases de dados da CAPES, não foram encontradas referências específicas de aplicação de controle preditivo MPC em nível de gasômetro com distribuição ótima e automática de combustíveis.

A sessão a seguir irá descrever brevemente a respeito da estratégia de controle de combustão clássica. Essa descrição se faz necessária para o entendimento da modificação dessa estratégia, descrita na seção 2.2 e que foi imprescindível para o funcionamento automatizado da distribuição de combustível.

\subsection{Controle de Combustão Clássico}

O controle eficiente de combustão requer a correspondência do combustível e ar em proporções estequiométricas. Uma prática comum é operar esses sistemas de combustão com valores ligeiramente superiores de ar para garantir a queima completa de todo o combustível. Entretanto, o excesso de ar reduz a temperatura de chama e a eficiência da transferência de calor [11].

Nos fornos e na caldeira da VBR envolvidos nesse trabalho, a estratégia de controle por duplo limite cruzado é usada para o controle da relação ar/combustível, conforme mostrado no diagrama simplificado da Figura 2 (a). Essa estratégia consiste no cálculo de janelas de operação (valores máximos e mínimos) para os setpoints de vazão de ar e gás. A janela de operação do ar de combustão depende do valor medido do gás e vice versa. A demanda energética calculada pelo controlador mestre (controlador de temperatura para fornos e de pressão para caldeiras) somente é passada para os controladores de vazão, se estiver dentro dessas janelas de operação. Evita-se, assim, o inconveniente de não serem atendidas as proporções desejadas quando há perturbações transitórias (o ar e o combustível podem ter diferentes dinâmicas, velocidades, e tempos para se acomodarem), ou quando há algum problema com os elementos de controle (falha, agarramento, etc.).

O combustível utilizado na VBR é o gás misto que consiste em uma mistura entre o GAF e o GN. O controle da proporção GAF/GN é realizado por uma estratégia de controle de razão simples conforme a Figura 2 (b).

* Contribuição técnica ao $18^{\circ}$ Seminário de Automação e TI Industrial, 23 a 26 de setembro de 2014, São Paulo, SP, Brasil. 


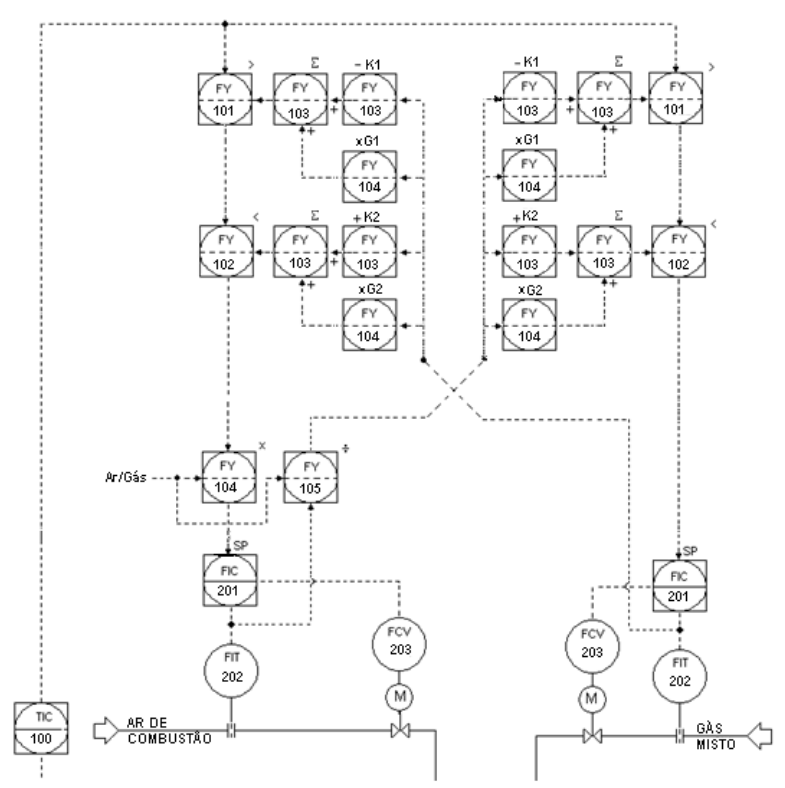

Figura 2. a) Estratégia duplo limite cruzado.

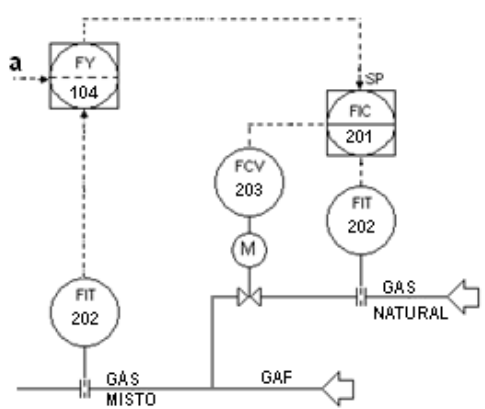

b) estratégia de controle de razão.

O valor da razão "a" é calculado conforme a Equação 1 . O objetivo é manter o $\mathrm{PCl}$ (poder calorífico inferior) do gás misto em um valor fixo. Além disso, o valor da relação ar/gás é ajustado de acordo com esse mesmo $\mathrm{PCl}$.

$$
a=\frac{P C I_{\text {desejado }}-P C I_{G A F}}{P C I_{G N}-P C I_{G A F}}
$$

\section{MATERIAIS E MÉTODOS}

O controlador MPC utilizado na VBR foi o DMC Plus da Aspentech Technology. O nome DMC (Dynamic Matrix Control) foi herança do nome dado ao controlador quando foi desenvolvido pelos engenheiros da Shell em 1973 [12].

Apesar de ser um software de mercado consolidado, há desafios consideráveis na implantação desse tipo de solução. Os maiores obstáculos são: (i) estabelecer uma coleta de dados robusta e de alta disponibilidade em distintas partes da usina; (ii) implementar as lógicas de controle regulatório para que os setpoints enviados pelo MPC sejam de fato seguido pelas malhas PID de forma robusta e sem muitos distúrbios para o processo e (iii) envolvimento e colaboração de diversas equipes de operação. A Figura 3 ilustra a hierarquia do MPC implantado na VBR.

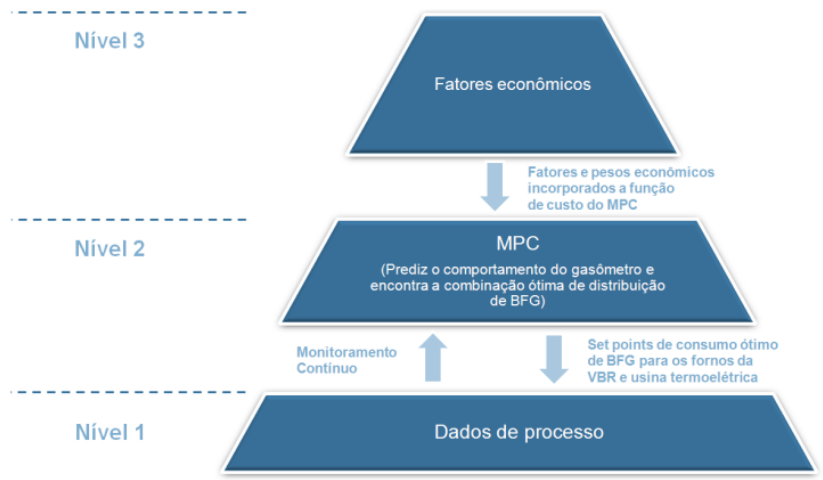

Figura 3. Hierarquia típica de um MPC.

* Contribuição técnica ao $18^{\circ}$ Seminário de Automação e TI Industrial, 23 a 26 de setembro de 2014, São Paulo, SP, Brasil. 


\subsection{Detalhes do Desenvolvimento do Controlador MPC}

As etapas e práticas típicas de um desenvolvimento de um MPC são bem conhecidas e difundidas. A Figura 4 mostra o passo a passo utilizado para 0 desenvolvimento na VBR.

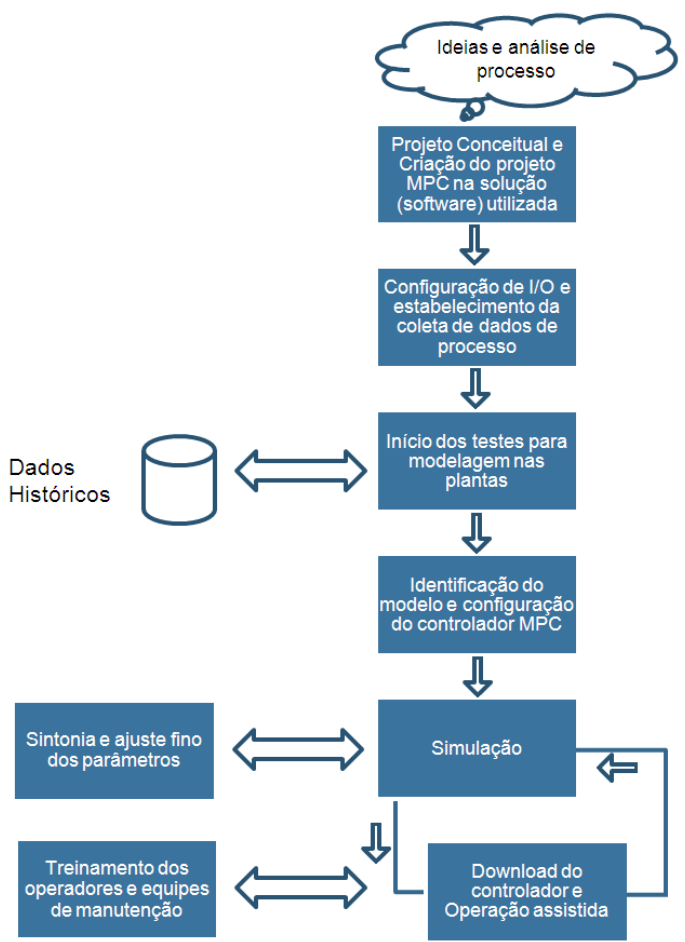

Figura 4. Fluxograma típico de um projeto de MPC. Fonte: Adaptado de Blevins [13].

Durante a etapa de modelagem matemática do nível do gasômetro, foi necessário levar em consideração todas as variáveis que influenciam no seu comportamento, conforme ilustra a Figura 5.

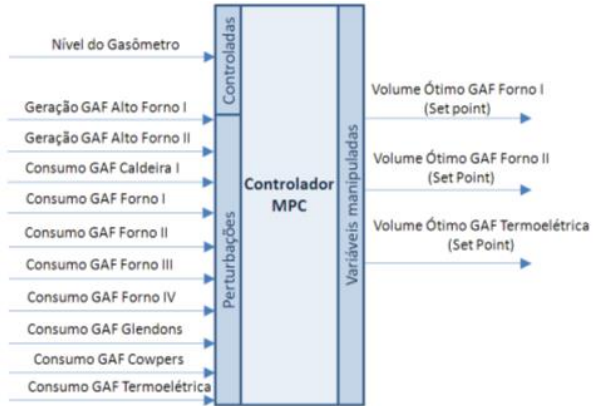

Figura 5. Estrutura da matriz de modelos multivariável adotada para o MPC. Fonte: VBR.

Os modelos utilizados para compor essa matriz, foram lineares, do tipo FIR (finite impulse response), onde as mudanças na saída de um processo são resultado de um somatório ponderado de mudanças passadas nas variáveis de entrada [9].

A matriz de modelos configurada para esse trabalho resultou em uma matriz de dimensão 11x3 onde há uma variável controlada (nível do gasômetro, CV), três variáveis manipuladas (setpoints de volume de GAF para os consumidores, MVs) e dez variáveis do tipo FeedForward (distúrbio).

* Contribuição técnica ao $18^{\circ}$ Seminário de Automação e TI Industrial, 23 a 26 de setembro de 2014, São Paulo, SP, Brasil. 
O modelo FIR, obtido por meio de dados históricos e conhecimento do processo, possui 30 coeficientes e um tempo de acomodação (time to steady state) de 5 minutos. Resultou em um controlador com ciclos de execução a cada 10s. Maiores detalhes da formulação matemática da matriz dinâmica, vide Cutler [12]. A Figura 6 mostra a resposta do modelo preditivo do nível do gasômetro.

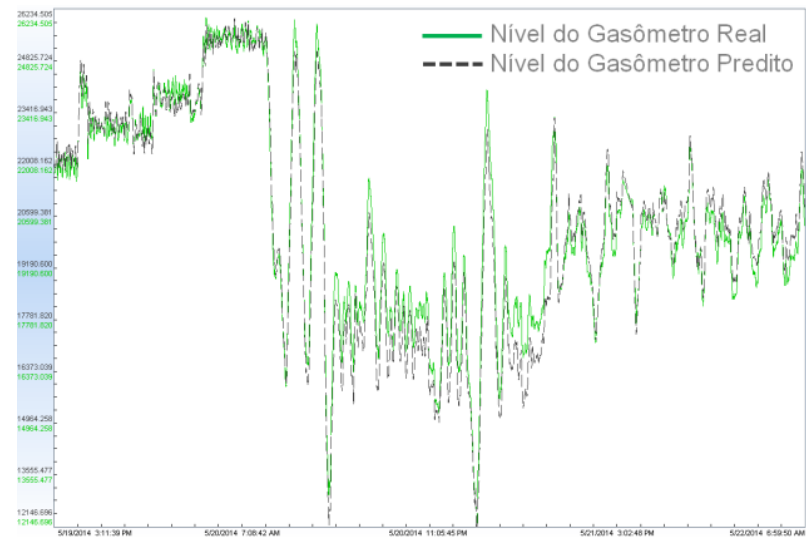

Figura 6. Resposta do modelo preditivo do gasômetro (3 dias de observação).

Após obter um modelo inicial a próxima etapa é configurar e ajustar os parâmetros do controlador para a otimização em estado estacionário e para a otimização dinâmica. Essa etapa é crucial para que o controlador realize exatamente sua função de controle preditivo minimizando uma função de custo. Em outras palavras, não importa o quão bom seja o modelo obtido até aqui, pois, caso as configurações desses módulos de otimização sejam negligenciados, o controlador terá um desempenho insatisfatório.

O módulo de otimização em estado estacionário nada mais é do que uma função de custo que será minimizada por um algoritmo de otimização linear (LP, linear programming) ou quadrática (QP, quadratic programming). Nesse artigo, o algoritmo utilizado para minimizar a função de custo é do tipo LP, conforme mostram a Equação 2 abaixo [14]:

$$
\phi=\text { Custo }_{1} * \Delta M V_{1 S S}+\text { Custo }_{2} * \Delta M V_{2 S S}+\ldots
$$

Após ajustar os parâmetros relativos à otimização em estado estacionário, os parâmetros para a otimização dinâmica necessitam de sintonia. A otimização dinâmica influencia em como o MPC irá aplicar os setpoints nas malhas PID de forma que o erro, tanto nas MVs como nas CVs, sejam mínimos e as restrições (limites) não sejam violadas. A Figura 7 exemplifica como a otimização estacionária e dinâmica funcionam na prática.

* Contribuição técnica ao $18^{\circ}$ Seminário de Automação e TI Industrial, 23 a 26 de setembro de 2014, São Paulo, SP, Brasil. 


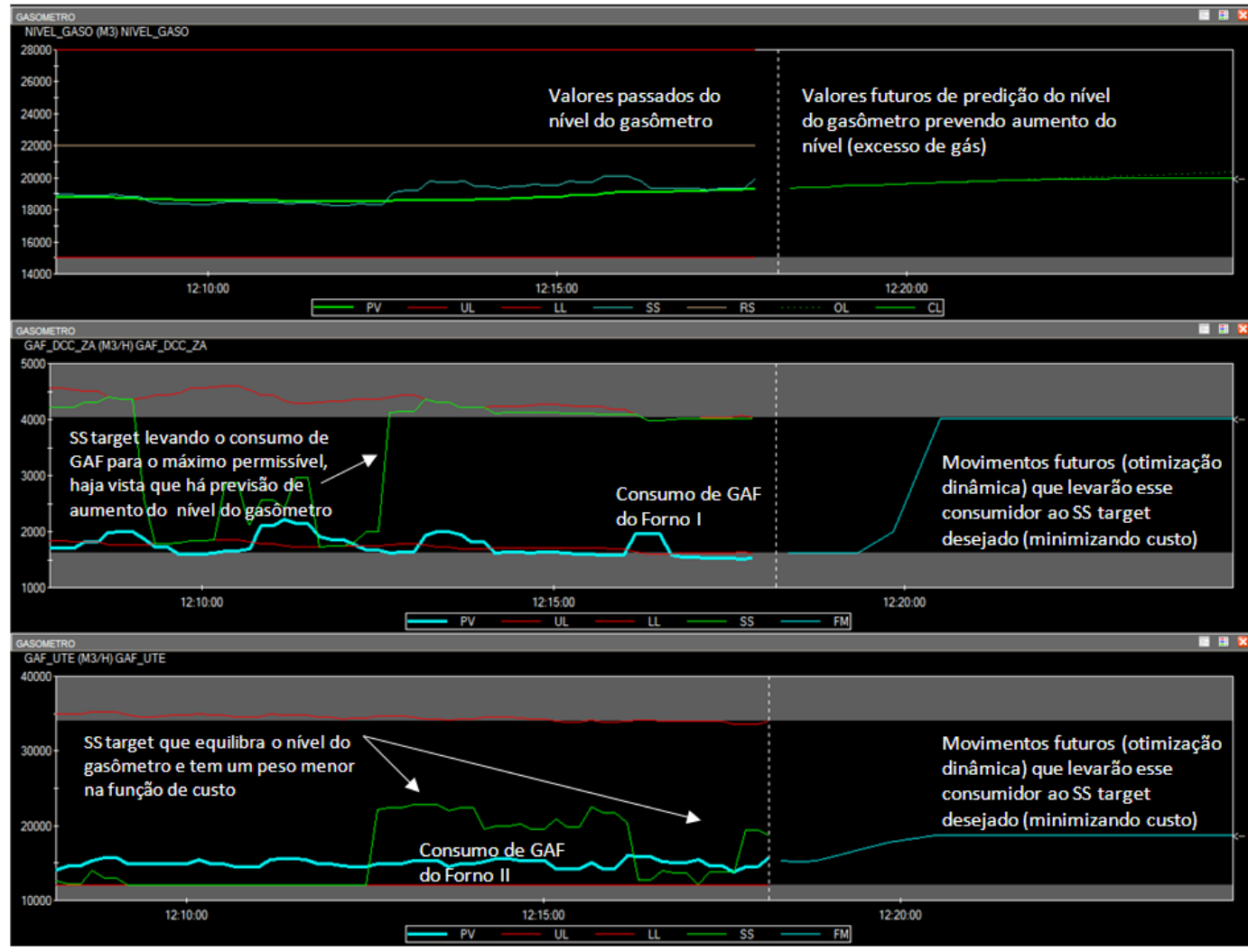

Legenda: PV (variável de processo); UL (limite superior); LL (limite inferior); SS (target em estado estacionário); RS (set point do gasômetro); OL (predição em malha aberta); CL (predição em malha fechada); FM (movimentos futuros ou otimização dinâmica).

Figura 7. Exemplo de como a otimização estacionária e dinâmica funcionam para o MPC implantado na VBR.

Na prática, o que acontece é que a parametrização inicial do controlador é feita por tentativa e erro, pois pouco ou quase nada se sabe do seu comportamento. Portanto, a próxima etapa, que é a de simulação, é fundamental para que se comece a ter um pouco mais de conhecimento sobre o impacto de cada um dos inúmeros parâmetros de ajuste e sintonia. Até mesmo o próprio fabricante relata em seu manual que muitos dos parâmetros são ajustados de forma empírica. A lição aprendida é que se deve gastar um bom e precioso tempo nessa etapa até que se tenha uma boa ideia de como o controlador poderá comportar durante a próxima etapa, que é a de colocá-lo em produção. Certificar-se de que os targets e as MVs estão respondendo de forma suave (a fim de causar pouco distúrbio no processo) e como o controlador se comporta com a CV perto dos limites operacionais é imprescindível para que se tenha segurança na hora de partir o MPC.

A etapa final, colocar o MPC em produção, é sem dúvida onde se deve prever a maior parte do tempo do projeto (2 a 3 meses no mínimo), haja vista a necessidade de envolvimento e treinamento dos operadores de cada equipamento que receberá os setpoints do MPC e, não obstante, o reajuste online dos parâmetros de sintonia feitos na etapa de simulação. O ideal é que as MVs sejam habilitadas de forma gradativa (uma de cada vez), a fim de mitigar incertezas e distúrbios para a planta.

Após a implementação do controlador, toda a documentação final do projeto foi elaborada (manual, procedimentos de backup, guia de sintonia e ajustes) de forma a facilitar a manutenção e sustentabilidade dos benefícios que foram obtidos com esse projeto.

\footnotetext{
* Contribuição técnica ao $18^{\circ}$ Seminário de Automação e TI Industrial, 23 a 26 de setembro de 2014,
} São Paulo, SP, Brasil. 


\subsection{Detalhes do Desenvolvimento do Controle nos Consumidores}

Para que os valores ótimos de consumo de GAF sejam seguidos automaticamente pelos consumidores é necessário alterar a estratégia clássica de controle de combustão descrita na seção 1.3.

O valor desejado de $\mathrm{PCI}$ não será mais fixo, conforme a Equação 1. O PCI deve ser variável, para permitir que a unidade siga o consumo desejado de GAF sem afetar 0 processo controlado. A Figura 8 mostra a estratégia alterada.

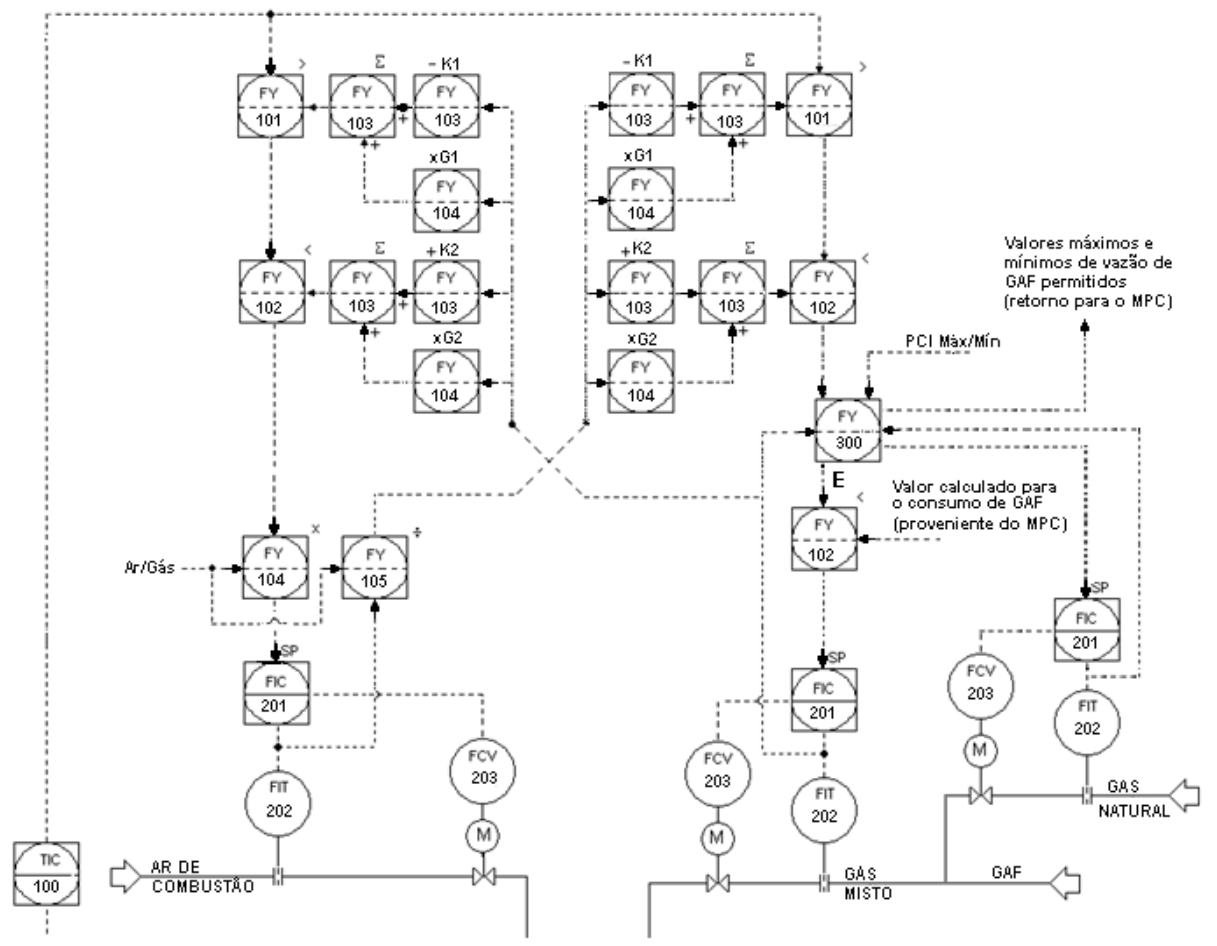

Figura 8. Estratégia de duplo limite cruzado alterada para receber o setpoint de vazão de GAF enviado pelo MPC.

Nota-se que na Figura 8 foram incluídos os blocos de cálculo FY300 e FY102. O bloco FY102 apenas limita o consumo de GAF ao valor desejado pelo controlador MPC. O bloco FY300 possui duas funções básicas. A primeira é o cálculo dos valores máximo e mínimo permitido de consumo de GAF na unidade em questão. Tal cálculo é feito conforme as Equações 3 e 4 . Os valores resultantes são informados para o controlador MPC com o objetivo de evitar o efeito de windup.

$$
\begin{aligned}
& V_{G A F_{-} M A X}=\frac{E}{P C I_{G A F}+P C I_{G N} \frac{\left(P C I_{G A F}-P C I_{M i n}\right)}{\left(P C I_{M i n}-P C I_{G N}\right)}} \\
& V_{G A F_{-} M I N}=\frac{E}{P C I_{G A F}+P C I_{G N} \frac{\left(P C I_{G A F}-P C I_{M a x}\right)}{\left(P C I_{M a x}-P C I_{G N}\right)}}
\end{aligned}
$$

Tais limites protegem a operação do processo controlado garantindo que a vazão necessária de GAF (E), calculada pelo duplo limite cruzado, seja atendida. A segunda função é o cálculo dos setpoints para cada controlador PID de vazão de combustível. Verifica-se, nessa etapa, a diferença entre a vazão necessária de GAF

* Contribuição técnica ao $18^{\circ}$ Seminário de Automação e TI Industrial, 23 a 26 de setembro de 2014, São Paulo, SP, Brasil. 
(E) e a vazão de GN (convertida para vazão equivalente de GAF). Com essa diferença, calcula-se o setpoint de GAF, conforme Equação 5.

$$
S_{P_{G A F}}=E-V_{G N} \frac{P_{G I_{G N}}}{P_{G_{G A F}}}+K
$$

Analogamente, é verificada a diferença entre a vazão necessária de GAF (E) e a vazão real de GAF, para calcular o setpoint de GN, conforme Equação 6.

$$
\mathbf{S P}_{\mathrm{GN}}=\mathbf{E}-\mathbf{V}_{\mathrm{GAF}}-\mathbf{K}
$$

Dessa forma, um combustível tenta complementar a energia que o outro não forneceu e o fator de transição $(K)$ força o sistema a maximizar o consumo de GAF em detrimento do consumo de GN. Quanto maior o valor do fator $K$ (valor dado em unidade de engenharia $\mathrm{m}^{3} / \mathrm{h}$ ), mais rápida é a substituição de um combustível pelo outro.

\section{RESULTADOS E DISCUSSÃO}

A Figura 9 apresenta o comportamento do nível do gasômetro e a abertura das tochas dos alto fornos antes e depois da implantação do controlador MPC. Observase que o nível do gasômetro (em vermelho) distancia-se do limite operacional (em azul) e que as tochas (em verde) reduzem drasticamente sua frequência de abertura.

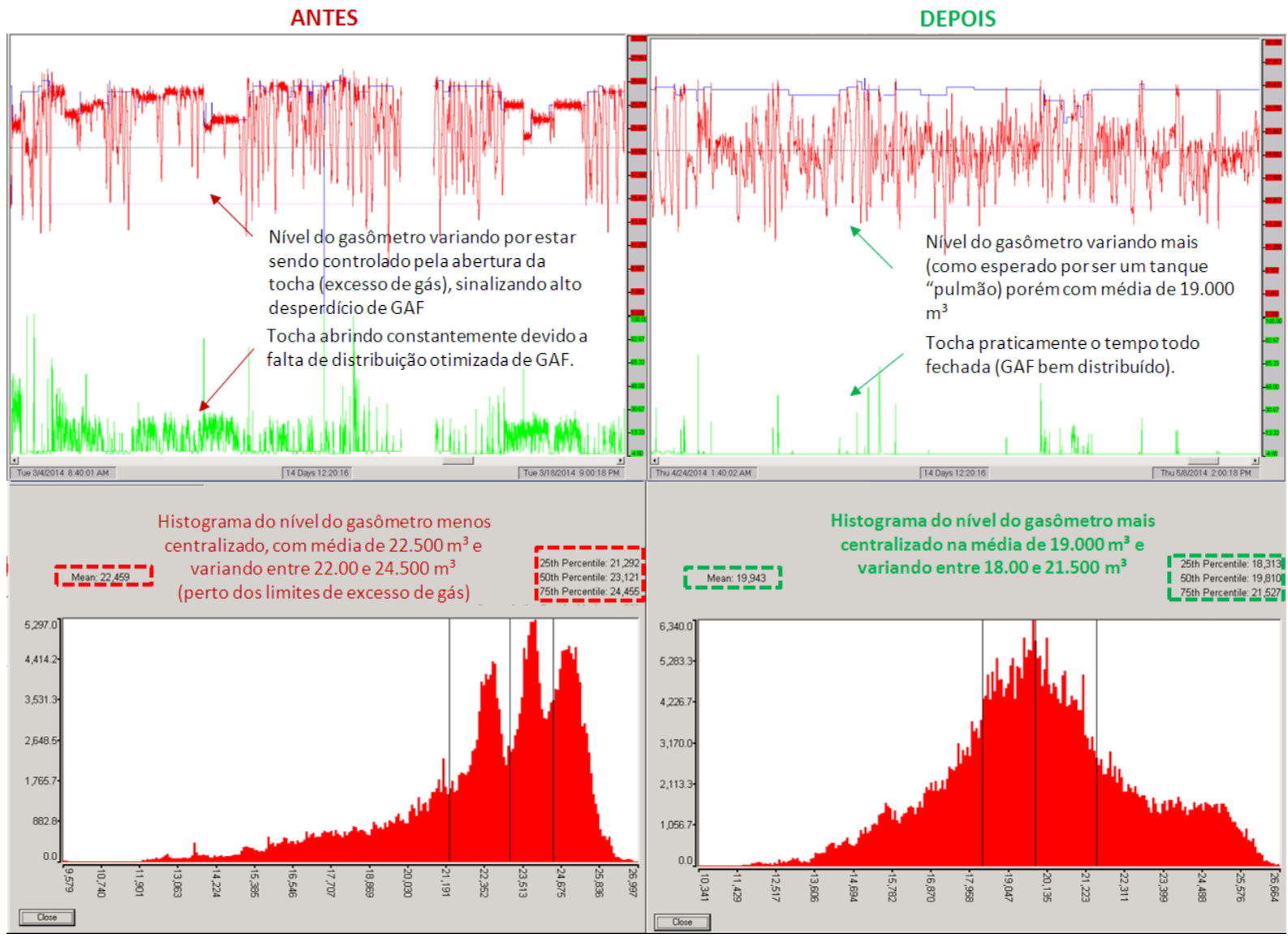

Figura 9. Comportamento do nível do gasômetro e abertura das tochas dos alto fornos da VBR.

* Contribuição técnica ao $18^{\circ}$ Seminário de Automação e TI Industrial, 23 a 26 de setembro de 2014, São Paulo, SP, Brasil. 
A implantação do controlador MPC na Vallourec Tubos do Brasil resultou em ganhos para a empresa, sendo eles:

- Ganhos financeiros relativos à redução de queima de GAF nas tochas dos alto fornos (maior uso de GAF e menor consumo de GN);

- Redução dos impactos ambientais devido à menor queima de combustível fóssil. O GAF é proveniente de alto fornos alimentados a carvão vegetal, produzido com madeira de reflorestamento;

- Melhor qualidade dos produtos produzidos, haja vista que a troca de combustível nos fornos passou a ser automática e suave;

- Redução de risco operacional do gasômetro, tendo em vista a diminuição da necessidade de intervenção humana no processo.

\subsection{Resultados Financeiros e Método de Apuração}

Os resultados de negócio foram apurados por meio da medição das perdas diárias de GAF nas tochas com relação à geração total de GAF nos altos fornos da VBR. Considera-se que cada metro cúbico não queimado nas tochas substituiu o volume equivalente de GN nos processos controlados.

A Figura 10 mostra a evolução das perdas. Houve uma redução considerável após 0 início da operação do controlador MPC, resultado deste trabalho. Os meses de Dezembro/2013, Janeiro/2014 e Fevereiro/2014 foram desconsiderados devido às paradas de produção das laminações e do alto forno 01.

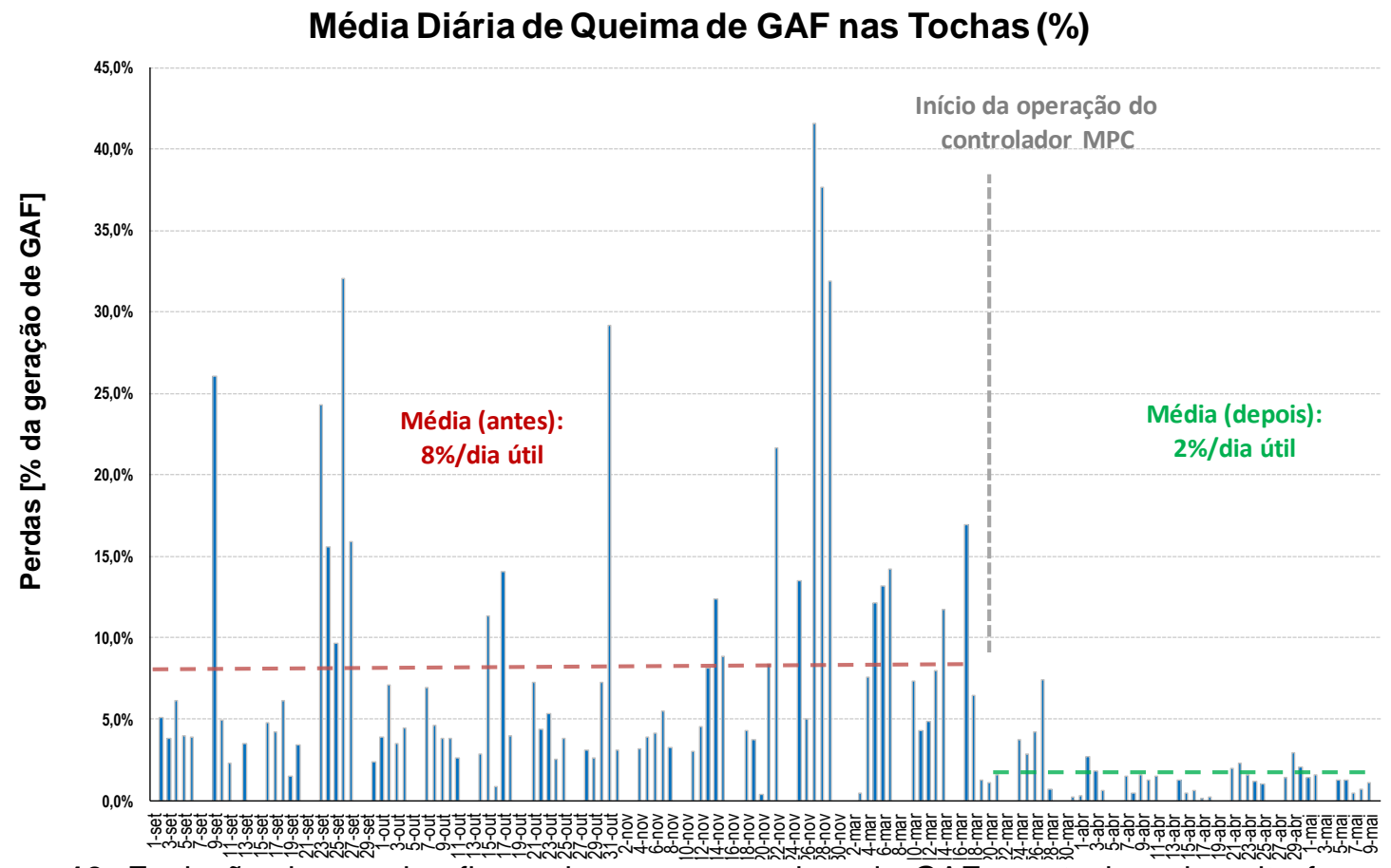

Figura 10. Evolução das perdas financeiras com a queima de GAF nas tochas dos alto fornos da VBR.

\section{CONCLUSÃO}

O desenvolvimento do controlador MPC para o controle preditivo do gasômetro e a distribuição otimizada e automática de combustível, apresentado nesse artigo, contribuiu para o aumento da eficiência energética da Vallourec Tubos do Brasil. A

* Contribuição técnica ao $18^{\circ}$ Seminário de Automação e TI Industrial, 23 a 26 de setembro de 2014, São Paulo, SP, Brasil. 
coleta de dados, o uso de modelos matemáticos e a determinação de valores otimizados de consumo de gás de alto forno em tempo real são procedimentos viáveis e podem ser aplicados na prática. Não se pode, no entanto, esquecer os ajustes e o bom desempenho dos controles regulatórios, afinal, é nesse nível do controle de processos que se realizam os resultados.

Esse trabalho é um incentivo para o aumento do número de aplicações de controladores avançados, em especial o MPC, no segmento minero metalúrgico.

\section{REFERÊNCIAS}

1 Kong H, Qi E, He S, Li G. MILP model for plant-wide optimal byproduct gas scheduling in iron and steel making industry. Journal of Iron and Steel Research. 2010; 17(7):3437.

2 Kong H, Qi E, Li H, Li G, Zhang X. An MILP model for optimization of byproduct gases in the integrated iron and steel plant. Applied Energy. 2010; 87:2156-2163.

3 Akimoto K, Sannomiya N, Nishikawa Y, Tsuda T. An Optimal Gas Supply for a Power Plant Using a Mixed Integer Programming Model. Automatica. 1991; 27(3):513-518.

4 Vieira CD, Carneiro MV, Rodrigues FM. Controle de Nível do Gasômetro por Substituição de Combustível na Termoelétrica. In: Associação Brasileira de Metalurgia, Materiais e Mineração. Anais do 34 Seminário de Balanços Energéticos Globais e Utilidades; 2013; Vitória, Brasil. São Paulo: ABM; 2013.

$5 \mathrm{Kim}$ JH, YI HS, Han C. A novel MILP model for plantwide multiperiod optimization of byproduct gas supply system in the iron and steel making process. Transactions IChemE. 2003; 81 (A):1015-1025.

$6 \mathrm{Kim} \mathrm{JH}, \mathrm{YI}$ HS, Han C. Plant-wide optimal byproduct gás distribution and holder level control in the iron and steel making process. Korean Journal of Chemical Engineering. 2003; 20(3):429-435.

7 Cardoso LN, Gonçalves VM, Takahashi HJ, Duarte CRM, Caminhas WM. Otimização da distribuição de combustíveis da Usiminas 2011 - Usina de Ipatinga. In: Associação Brasileira de Metalurgia, Materiais e Mineração. Anais do 33 Seminário de Energia e Utilidades; 2012; Belo Horizonte, Brasil. São Paulo: ABM; 2012. p. 120-131.

8 Zhang X., Zhao J. Wang W., Cong L., Feng W. An optimal method for prediction and adjustment on byproduct gas holder in steel industry. Expert systems with applications. 2011; 38:4588-4599.

9 Qin SJ, Badgwell TA. A survey of industrial model predictive control technology. Control Engineering Practice. 2003; 11:733-764.

10 Qin SJ, Badgwell TA. Industrial Model Predictive Control. An Updated Overview. 2002 [acesso em 01 mai. 2014]; Disponível em: http://nt.ntnu.no/users/skoge/presentation/ mpc_badgwell/mpc_survey_handout.pdf.

11 Shinskey G. Process Control Systems: Applications, Design and Tuning. 4를 Ed. New York: McGraw-Hill; 1996.

12 Cutler CR, Ramaker BL. Dynamic matrix control - a computer control algorithm. In: AICHE national meeting, Houston, TX, 1979.

13 Blevins T, Wojsznis WK, Nixon M. Advanced Control Foundation: Tools, Techniques and Applications. Durham: ISA; 2013.

14 Aspentech Technology. Introduction to Advanced Process Using DMCPlus. Aspentech Customer Education Training Manual. 2013.

* Contribuição técnica ao $18^{\circ}$ Seminário de Automação e TI Industrial, 23 a 26 de setembro de 2014, São Paulo, SP, Brasil. 